\title{
Novel MIMO Communication Scheme for Enhanced Indoor Performance in Distributed Antenna Systems
}

\author{
Bong Youl Cho $\cdot$ Jin Young Kim
}

\begin{abstract}
Multiple input multiple output (MIMO) has been considered one of the key enablers of broadband wireless communications. The indoor environment is known to be favorable to ensure both high rank property and high signal-to-interference/noise ratio (SINR) to fully exploit MIMO spatial multiplexing (SM) gain. In this paper, we describe several practical deployment cases where repeater links (or relay links), such as those present with an indoor distributed antenna system (DAS), can act as keyholes to degenerate the rank property of MIMO communications. In this case, we cannot exploit MIMO SM gain in indoor environment. We propose a novel MIMO communication scheme which uses simple converter in the devices in repeater links to resolve the rank degeneration issue and to ensure MIMO SM gain in highly MIMO-favorable indoor environment. MIMO SM is possible over the indoor DAS with single cable line through use of simple converters, which enables practical deployment in real fields.
\end{abstract}

Key words : MIMO, Keyhole, Repeater, Relay, DAS.

\section{Introduction}

MIMO antenna technology, where multiple antennas are used on the transmitter and receiver sides, has been considered one of the key enablers of broadband wireless communications. A key feature of MIMO systems is the ability to turn multipath propagation into a benefit for users by multiplying transfer rates $[1,2]$. This multiplexing capability is enabled by the fact that a scattering environment makes the signal from every individual transmit antenna deliverable through a distinct spatial signature for reception.

The high spectral efficiency in MIMO communications is reduced if the signals arriving at receivers are correlated. Correlation between antennas may be reduced in actual deployments by separating antennas spatially. However, it has been shown that low correlation is not a guarantee of high capacity. Chizhik et al. [3, 4] and Gesbert et al. [5] showed what they called a "keyhole" (Chizhik) or "pinhole" (Gesbert) effect; we will call it "keyhole" or "keyhole effect" from here forward. This keyhole effect exhibits a case where the channel matrix has low correlation, yielding diversity gain, but has a single degree of freedom that doesn't yield multiplexing gain. In additions, SM may not be the optimum way to use MIMO configuration in practical MIMO systems if channel SINR is not high enough to ensure reliable MIMO decoding. In this case, diversity at both ends (ie, Tx di- versity at transmitter and $\mathrm{Rx}$ diversity at receiver) can be better way to use MIMO system configuration. Indoor environment is known to be favorable to ensure both high rank property and high SINR to fully exploit MIMO SM gain [6, 7], and that is why IEEE $802.11 \mathrm{n}$ adopted MIMO in a very early stage [8].

In cellular communications, operators need to guarantee good coverage performance. Repeaters (or relays) have been considered cost-effective in providing good coverage that complements conventional macro-base station (BS). There have been studies on MIMO communications with relays. In [9], upper bounds and lower bounds of channel capacity are shown in MIMO relay channels. Most of studies examined cooperation, where there is a signal path from original BS to mobile station (MS) as well as a path through relay. Or, there is research about MIMO communications with relays which are equipped with multiple antennas at both transmit and receive ends to reserve the rank of overall link [10].

However, in practice, there can be a case where a relay has single input single output (SISO) capability, which means the relay has only one receive antenna and one transmit antenna, and there is no direct signal path from original BS to MS, which means an isolated area. This case is often observed in real deployments:

1) The underground basement can be covered by amplify-and-forward relay (or RF repeater) that receives signals from BS with receive antenna lo-

Manuscript received June 21, 2010 ; revised September 16, 2010. (ID No. 20100621-020J)

Department of Electronics Convergence Engineering, Kwangwoon University, Seoul, Korea.

Corresponding Author: Jin Young Kim (e-mail : jinyoung@kw.ac.kr) 
cated outdoors and serves the basement area with transmit antenna located in the basement.

2) The indoor floors of tall buildings can be covered by indoor DAS which is comprised of distribution network with cable(s) and remote units (or RF units) (RU) in each floor to serve the targeted area [11].

Please note that the above DAS system is different from MIMO-DAS system which is also known as co-operative multi-point transmission and reception (COMP), or virtual MIMO [12]. In the above examples, RF repeater and indoor DAS can be acting as keyholes if these are not MIMO-capable. The problem to be solved is that the above repeaters provide coverage mostly for indoor environment, which is purportedly very good for obtaining MIMO SM gain. But, due to the keyhole effect by the repeater itself, it is impossible to get any SM gain in this environment. Several studies were performed on MIMO repeater system where there is a keyhole effect. Tsuruta et al. estimated the probability density function (PDF) of eigenvalues in MIMO multi keyhole environment [13].

We focus on indoor DAS in this paper and propose a novel scheme to enable MIMO communications that can exploit SM gain even in the case where single cable line is used in the distribution network [14]. Since indoor channel characteristics are generally favorable for MIMO SM, significant capacity enhancement as well as coverage enhancement is expected by using the proposed scheme while keeping deployment cost reasonable for operators.

This paper is organized as follows. First, MIMO communications system and keyhole effect are described in section $\Pi$. We review the conventional scheme to enable MIMO SM in indoor DAS in section III, and explain the proposed scheme in section IV. The performance enhancement possible through use of the proposed scheme is discussed in section $\mathrm{V}$, followed by conclusions in section VI.

\section{II . MIMO System and Keyhole}

Let's assume $2 \times 2$ MIMO communications as in Fig. 1 where we want to deliver two different signal streams simultaneously. In Fig. 1, transmitter can be BS and receiver can be MS in downlink, and transmitter can be MS and receiver can be BS in uplink. The data rate increase by SM gain is not always possible, but rather is dependent on the characteristics of the channel matrix, denoted as $\mathbf{H}$ in Fig. 1. This can be described as follows.

The order of data rate increase $\leq$ rank $(\mathbf{H}) \leq \min \left(N_{T X}, N_{R X}\right)$

In cellular communications, repeaters can be used as

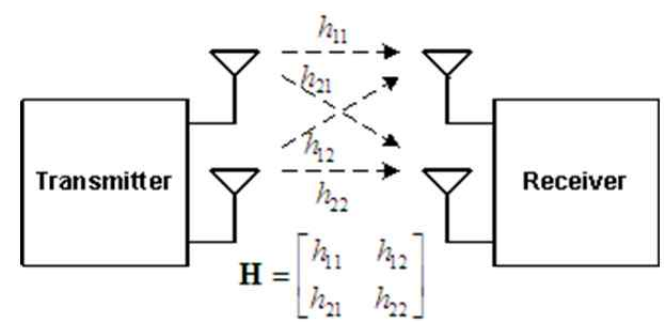

Fig. 1. Example of a MIMO system where there are 2 transmit antennas $\left(N_{T X}=2\right)$ in transmitter side and 2 receive antennas $\left(N_{R X}=2\right)$ in receiver side.

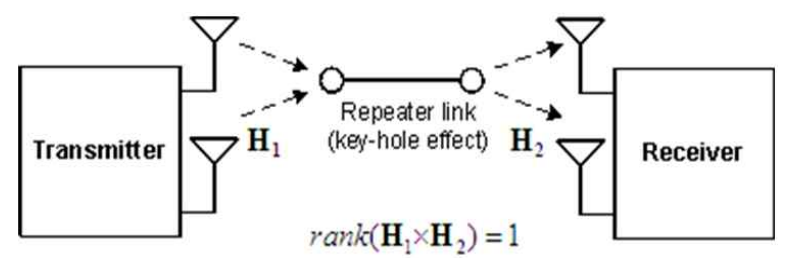

Fig. 2. Example system where the rank of overall link is 1 due to the keyhole effect.

a cost-effective solution to enhance coverage. When repeaters are introduced in MIMO communications, the rank property should be preserved in overall link if we want to get MIMO SM gain. If repeaters are not MIMOcapable and there is no direct signal path between BS and MS, these repeaters can act as keyholes and the rank of overall link is reduced to 1. Fig. 2 shows the keyhole effect generated by SISO repeater.

\section{Conventional Scheme}

Among various deployment scenarios with repeaters, here we focus on indoor DAS used to provide indoor coverage especially for tall buildings where down-tilted macro BS located below the high floors of tall buildings cannot provide coverage to the targeted area. Fig. 3 shows one example deployment of DAS for indoor coverage in MIMO communications. Since we want to preserve the rank property of overall link as 2 to get SM gain, two parallel cable lines are used between BS and RUs. Each RU is equipped with two transmit antennas. Therefore, it can be said that indoor DAS in Fig. 3 is MIMO-capable, and two signal streams can be delivered from BS to MSs in each floor depending on the exact channel realization between RUs and MSs.

However, this scheme can be very problematic in practice. The cable network is the governing factor in indoor DAS cost structure, so using 2 parallel cable lines (or multiple parallel cable lines in more generalized MIMO) can significantly increase the overall system deployment cost. Furthermore, it is sometimes not possible in practice to deploy additional cable line(s) to existing indoor 


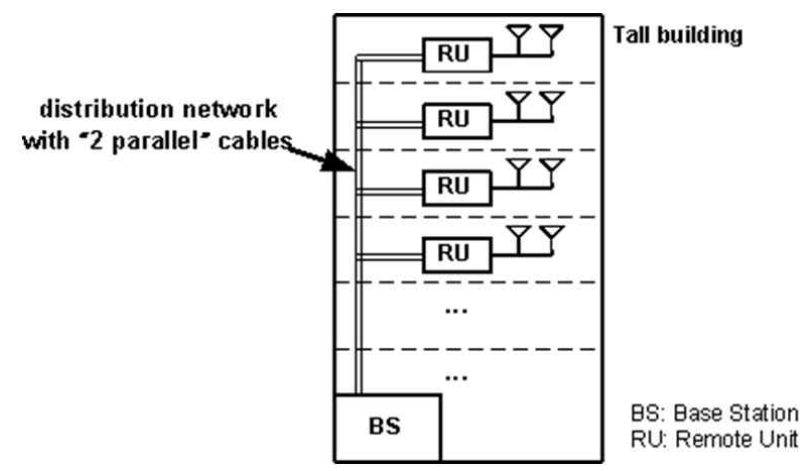

Fig. 3. Example of the conventional scheme with DAS for indoor coverage in MIMO communications where 2 parallel cable lines are used in the distribution network.

DAS due to building owners' resistance to building disruption. Most tall buildings are already covered by legacy communication systems such as GSM, WCDMA, cdma 2000, and EVDO by using indoor DAS, and existing indoor DAS is SISO-capable since those legacy systems are not using MIMO technology in real deployment yet.

\section{Proposed Scheme}

Fig. 4 shows one example deployment scenario of DAS for indoor coverage in MIMO communications with proposed scheme. In the proposed scheme, a single cable line is used in the distribution network as we put a converter block in BS and RU, respectively.

Fig. 5 shows the converter block in the proposed scheme. Converter block simply converts multiple parallel data streams in MIMO antenna domain into parallel data streams in another domain, and vice-versa. The scheme for data stream conversion can be frequency division multiplexing (FDM), whereby multiple parallel data streams in the MIMO antenna domain at the same time/frequency are converted into multiple parallel data streams in frequency domain. In this plan, each data stream is located at different frequencies for independent delivery. This is possible because we are using cable line (or equivalent media), which is isolated transmission media. However the converting schemes are not limited to FDM. For example, time division multiplexing (TDM) can be used where multiple parallel data streams in antenna domain at the same time/frequency are being converted into multiple parallel data streams in time domain where each data stream is delivered at different time at the same frequency. This is also possible because we are using cable line (or equivalent media), whereas the data rate in wireline can generally be much higher than that in wireless. We can deliver multiple parallel data streams (e.g., 2 parallel data streams) in MIMO antenna domain seria-

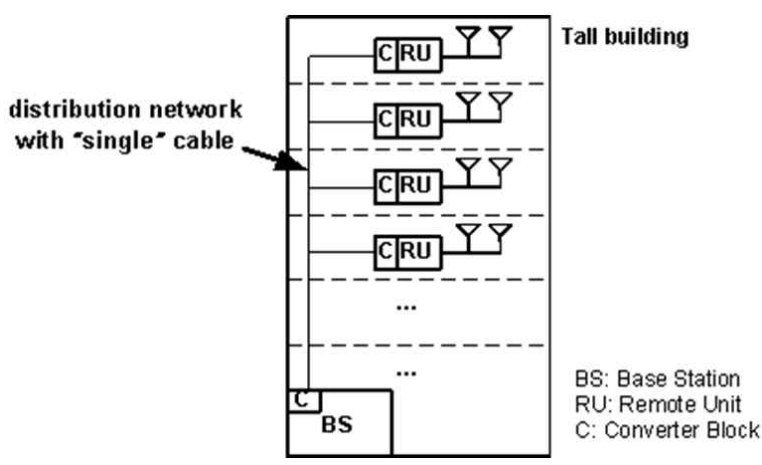

Fig. 4. Example of the proposed scheme with DAS for indoor coverage in MIMO communications where single cable line is used in the distribution network.

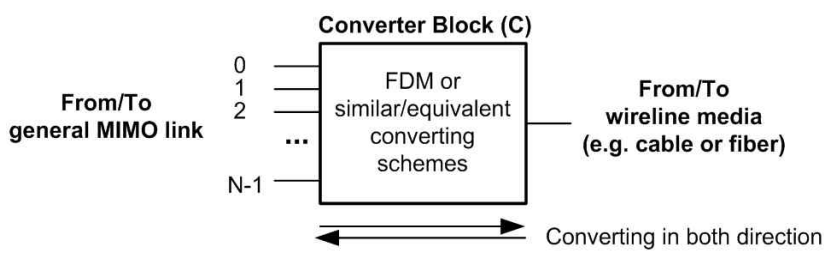

Fig. 5. Converter block which converts MIMO parallel data streams into parallel independent streams in other domain, and vice-versa.

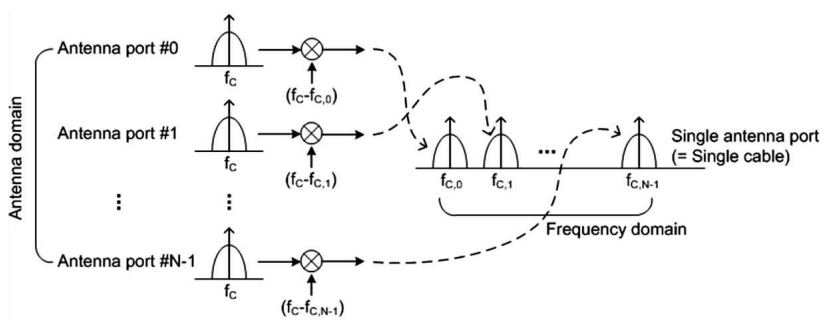

Fig. 6. Example converter block where FDM is used.

lly with higher transmission rate (e.g., 2 times faster) in wireline TDM case within the same time duration. Fig. 6 shows the example converter block where FDM is used.

The similar work has been done to enable MIMO communications over single cable line, but a highly echoic chamber was used in the previous works [15], which is different from the proposed scheme in this paper.

\section{Performance Enhancement}

In order to exploit SM gain in MIMO communications, the following requirements should be fulfilled. Low correlation and high rank property should be guaranteed in the channel matrix. At the same time, channel SINR should be high enough for the reliable decoding.

Fig. 7 shows performance comparison in terms of the spectral efficiency between $2 \times 2$ MIMO SM, $2 \times 2$ MIMO 


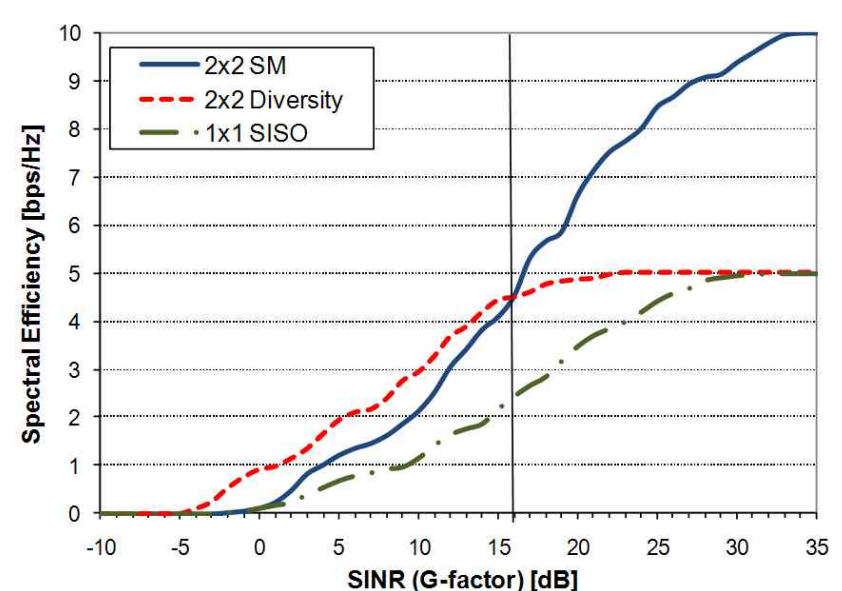

Fig. 7. Performance of $2 \times 2$ MIMO SM, $2 \times 2$ MIMO diversity, and $1 \times 1$ SISO in a typical environment.

diversity, and $1 \times 1$ SISO versus SINR values in a typical environment with the following parameters and conditions:

- IEEE 802.16e WiMAX system with $10 \mathrm{MHz}$ system bandwidth is considered.

- Pedestrian B channel with $3 \mathrm{~km} / \mathrm{h}$ mobility is assumed.

- 7 modulation and coding schemes (MCS) are considered: QPSK 1/2, QPSK 3/4, 16QAM 1/2, 64 QAM 1/2, 64QAM 2/3, 64QAM 3/4, and 64QAM $5 / 6$.

- Suboptimal maximum likelihood detector (MLD) is used for MIMO decoding.

- Tx/Rx spatial correlation of 0.5 is assumed for MIMO channel.

As in Fig. 7, MIMO SM outperforms MIMO diversity where enough SINR, for example, greater than 15 16 $\mathrm{dB}$ in this figure, is maintained for reliable decoding.

Fig. 8 shows the SINR distribution of a typical indoor

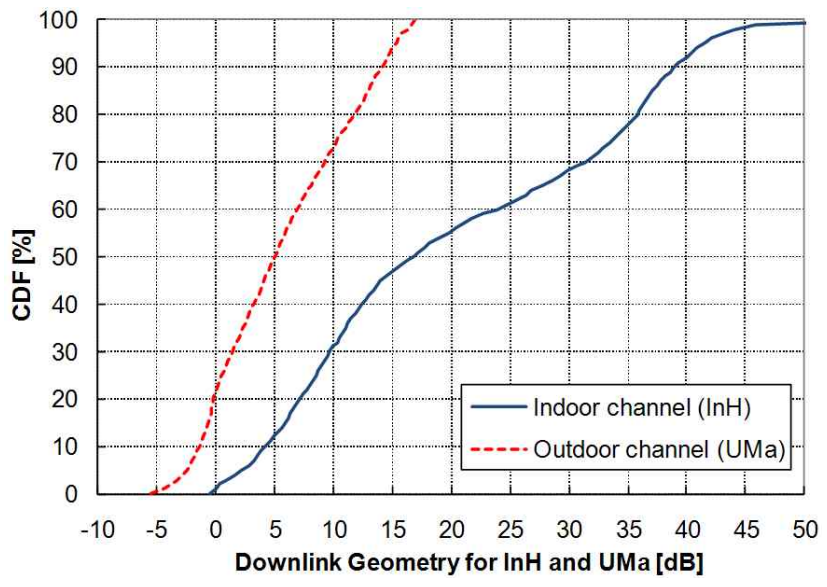

Fig. 8. SINR distributions for a typical indoor and outdoor channel.

channel and that of a typical outdoor channel from IMTAdvanced evaluation methodology document by ITU-R [16]. The detailed deployment scenarios for the indoor channel and the outdoor channel are summarized in Table 1. It is shown in Fig. 8 that the probability of having enough SINR where MIMO SM outperforms other schemes is much higher for the indoor channel than for the outdoor channel. The probability of having SINR more than $15 \mathrm{~dB}$ is greater than $50 \%$ for an indoor hotspot channel, while it is only about $5 \%$ in an urban macro-cell environment.

Seeing the characteristics of indoor channels, it will be a considerable loss in overall system capacity if we cannot use MIMO SM for MSs located in indoor floors, indoor basements, and other places where repeaters are used for coverage fulfillment. Therefore, the proposed scheme is expected to enhance system capacity significantly in addition to coverage extension by exploiting MIMO SM gain in many places, and especially in in-

Table 1. Deployment scenarios for indoor channel and outdoor channel in Fig. 8.

\begin{tabular}{|c|c|c|}
\hline Deployment scenario & $\begin{array}{l}\text { Indoor channel } \\
\text { (Indoor hotspot, InH) }\end{array}$ & $\begin{array}{c}\text { Outdoor channel } \\
\text { (Urban macro-cell, UMa) }\end{array}$ \\
\hline BS antenna height & $6 \mathrm{~m}$, mounted on ceiling & $25 \mathrm{~m}$, above rooftop \\
\hline Total BS transmit power & $21 \mathrm{dBm}$ & $49 \mathrm{dBm}$ \\
\hline $\begin{array}{l}\text { Minimum distance between MS and serving } \\
\text { cell }\end{array}$ & $\geq 3 \mathrm{~m}$ & $\geq 25 \mathrm{~m}$ \\
\hline Carrier frequency & $3.4 \mathrm{GHz}$ & $2 \mathrm{GHz}$ \\
\hline Layout & $\begin{array}{l}\text { Indoor floor. } \\
\text { Two sites located one floor of a building } \\
\text { contains } 16 \text { rooms of } 15 \times 15 \mathrm{~m} \text { and a } \\
\text { long hall of } 120 \times 20 \mathrm{~m} \text {. }\end{array}$ & $\begin{array}{l}\text { Hexagonal grid. } \\
\text { A wrap-around configuration of } 19 \text { sites, } \\
\text { each of } 3 \text { cells. }\end{array}$ \\
\hline Inter-site distance & $60 \mathrm{~m}$ & $500 \mathrm{~m}$ \\
\hline User distribution & $\begin{array}{l}\text { Randomly and uniformly distributed over } \\
\text { area }\end{array}$ & $\begin{array}{l}\text { Randomly and uniformly distributed over } \\
\text { area }\end{array}$ \\
\hline
\end{tabular}


Table 2. Average cell throughput per case.

\begin{tabular}{|c|l|c|}
\hline \multirow{5}{*}{ Environment } & \multicolumn{1}{|c|}{ Case } & $\begin{array}{c}\text { Average cell } \\
\text { spectral } \\
\text { efficiency } \\
{[\mathrm{bps} / \mathrm{Hz}]}\end{array}$ \\
\hline \multirow{4}{*}{ Indoor } & Only MIMO diversity is used & 3.79 \\
\cline { 2 - 3 } & Only MIMO SM is used & 5.51 \\
\cline { 2 - 3 } & MIMO AMS is used & 5.83 \\
\hline \multirow{3}{*}{ Outdoor } & Only MIMO diversity is used & 2.09 \\
\cline { 2 - 3 } & Only MIMO SM is used & 1.51 \\
\cline { 2 - 3 } & MIMO AMS is used & 2.11 \\
\hline
\end{tabular}

door locations.

Table 2 summarizes performance enhancement by enabling MIMO SM based on the results from Fig. 7 and Fig. 8. If the rank of overall link is 1 due to SISO-capable repeaters in the link, we have no choice but to use MIMO diversity all the time and we get $3.79 \mathrm{bps} / \mathrm{Hz}$ as average cell spectral efficiency in indoor environment and $2.09 \mathrm{bps} / \mathrm{Hz}$ in outdoor environment, respectively. If the rank of overall link is 2 thanks to MIMO-capable repeaters in the link, we can use MIMO SM as well depending on the channel characteristics. Obviously, using MIMO SM for all SINR regions is not the optimal scheme since MIMO SM underperforms MIMO diversity in low SINR region because decoding can be unreliable. Therefore, adaptive MIMO switching (AMS) is generally used in MIMO communications where BS adaptively switches MIMO mode between diversity and multiplexing depending on the channel characteristics (e.g., rank and SINR) $[17,18]$. Since AMS is not the main topic in this paper, we use a very simple switching algorithm to get the results in Table 2 where $\mathrm{SM}$ is used when channel SINR is greater than $15 \mathrm{~dB}$ and diversity is used otherwise. Huge performance enhancement is seen in Table 2 for the indoor environment when we can use SM as well as diversity in the link. Table 2 shows that spectral efficiency increases from $3.79 \mathrm{bps} / \mathrm{Hz}$ to $5.83 \mathrm{bps} / \mathrm{Hz}$, approximately a $54 \%$ capacity increase.

However, as described in section III, it can be very challenging to enable MIMO SM link in indoor DAS with conventional scheme although the capacity gain is huge. On the other hand, the proposed scheme enables MIMO SM link in a cost-effective manner.

Table 3 from ABI research shows that in-building wireless systems will grow significantly in the future since broadband wireless access systems such as WiMAX and LTE will require better indoor performance [19]. Almost all types of in-building wireless systems in Table 3 (e.g., distributed repeaters and active/passive DAS) require cable or wireline distribution network. In additions, ABI research [19] also says that building disruption can occur in active/passive DAS system and this issue makes the deployment of additional cable lines for MIMO SM enablement more difficult. Therefore, we believe that the proposed scheme in this paper can be a solution which solves MIMO keyhole effect in a very practical way in real fields.

\section{Conclusions}

MIMO systems can utilize multipath propagation in wireless channel into a benefit for users by multiplying transfer rates. The high spectral efficiency of MIMO systems is reduced if there is high correlation and/or keyhole effect in a channel matrix. Several practical deployment cases, such as indoor DAS for high floors in tall buildings, exhibit the keyhole effect if indoor DAS is not MIMO-capable.

Making indoor DAS MIMO-capable is sometimes not economically viable or not possible in practice. In this paper, a novel scheme has been proposed which enables MIMO SM communications in a very practical way, even over indoor DAS with single cable. Since indoor channel generally exhibits favorable characteristics for MIMO SM, the proposed scheme is expected to enhance system capacity significantly in a cost-effective manner.

This research has been supported by Research Fund by Small and Medium Business Administration in 2010. (Contract No. 00042462)

Table 3. In-building wireless systems deployment revenue by system type (\$ millions) [19].

\begin{tabular}{|l|c|c|c|c|c|c|}
\hline System type & 2009 & 2010 & 2011 & 2012 & 2013 & 2014 \\
\hline Distributed repeaters & 176 & 231 & 295 & 377 & 480 & 604 \\
\hline Active DAS & 2,693 & 3,451 & 4,423 & 5,661 & 7,230 & 9,244 \\
\hline Passive DAS & 1,940 & 2,395 & 2,954 & 3,637 & 4,468 & 5,490 \\
\hline Distributed radios & 0 & 1 & 12 & 31 & 59 & 108 \\
\hline Total & 4,809 & 6,078 & 7,685 & 9,705 & 12,237 & 15,445 \\
\hline
\end{tabular}




\section{References}

[1] G. J. Foschini, "Layered space-time architecture for wireless communication in a fading environment when using multielement antennas," Bell Labs Tech. J., pp. 41-59, 1996.

[2] E. Telatar, "Capacity of multiantenna Gaussian channels," AT \& T Bell Laboratories, Tech. Memo., Jun. 1995.

[3] D. Chizhik, G. Foschini, and R. A. Valenzuela, "Capacities of multielement transmit and receive antennas: Correlations and keyholes," Electron. Lett., vol. 36, issue 13, pp. 1099-1100, 2000.

[4] D. Chizhik, G. J. Foschini, M. J. Gans, and R. A. Valenzuela, "Keyholes, correlations, and capacities of multielement transmit and receive antennas," IEEE Trans. Wireless Commun., vol. 1, issue 2, pp. 361368, Apr. 2002.

[5] D. Gesbert, H. Bolcskei, D. Gore, and A. Paulraj, "Outdoor MIMO wireless channels: Models and performance prediction," IEEE Trans. Commun., vol. 50, issue 12, pp. 1926-1934, Dec. 2002.

[6] P. L. Kafle, A. Intarapanich, A. B. Sesay, J. McRory, and R. T. J. Davies, "Spatial correlation and capacity measurements for wideband MIMO channels in indoor office environment," IEEE Trans. Wireless Commun., vol. 7, no. 5, pp. 1560-1571, May 2008.

[7] R. Stridh, K. Yu, B. Ottersten, and P. Karlsson, "MIMO channel capacity and modeling issues on a measured indoor radio channel at $5.8 \mathrm{GHz}$," IEEE Trans. Wireless Commun., vol. 4, no. 3, pp. 895903, May 2005.

[8] IEEE Std 802.11n-2009, IEEE standard for information technology - Telecommunications and information exchange between systems - Local and metropolitan area networks - Specific requirements Part 11: Wireless LAN Medium Access Control (MAC) and Physical Layer (PHY) Specifications Amendment 5: Enhancements for Higher Throughput.

[9] B. Wang, J. Zhang, and A. Host-Madsen, "On the capacity of MIMO relay channels," IEEE Trans. Infor- mation Theory, vol. 51, no. 1, pp. 29-43, Jan. 2005.

[10] X. Tang, Y. Hua, "Optimal design of non-regenerative MIMO wireless relays," IEEE Trans. Wireless Commun., vol. 6, no. 4, pp. 1398-1407, Apr. 2007.

[11] A. A. M. Saleh, A. J. Rustako, and R. S. Roman, "Distributed antennas for indoor radio communication," IEEE Trans. Commun., vol. 35, issue 12, pp. 1245-1251, Dec. 1987.

[12] P. Marsch, S. Khattak, and G. Fettweis, "A framework for determining realistic capacity bounds for distributed antenna systems," IEEE Information Theory Workshop, pp. 571-575, Oct. 2006.

[13] M. Tsuruta, T. Taniguchi, and Y. Karasawa, "On statistical distribution of eigenvalues of channel correlation matrix in MIMO multi-keyhole environment," IEICE Trans Commun., Sep. 2007.

[14] B. Y. Cho, S. I. Seo, and J. Y. Kim, "Practical scheme to enable MIMO communications in distributed antenna systems for efficient indoor coverage," in Proceedings of the 2009 IEEE International Symposium on Communication and Information Technology (IEEE ISCIT '09), pp. 25-28, Incheon, Korea, Sep. 2009.

[15] E. Weinstein, "Providing a high speed data modem based on MIMO technology using a cable or single antenna," US Patent No.: US 7.346.040 B2, Issued on Mar. 2008.

[16] Report ITU-R M.2135, "Guidelines for evaluation of radio interface technologies for IMT-Advanced," 2008.

[17] Robert W. Heath, Jr., Arogyaswami J. Paulraj, "Switching between diversity and multiplexing in MIMO systems," IEEE Trans. Commun., vol. 53, no. 6, pp. 962-968, Jun. 2005.

[18] S. Choi, Y. Ko, and E. J. Powers, "Optimization of switched MIMO systems over Rayleigh fading channels," IEEE Trans. Vehicular Technol., vol. 56, no. 1, pp. 103-114, Jan. 2007.

[19] ABI research, "In-building wireless systems: Passive and active DAS, repeaters, picocells, and femtocells," 3Q 2009. 


\section{Bong Youl Cho}

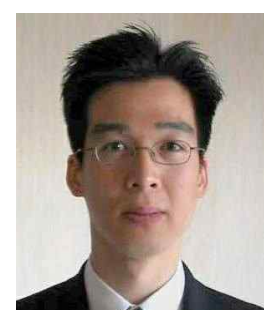

received the B.Sc. and M.Sc. in electrical engineering from Seoul National University (SNU), Seoul, Korea, in 1997 and 1999, respectively. In 1999, he joined radio research division at KTF in Seoul, Korea, and worked on the physical and MAC layers of IS-95 A/B and WCDMA. In 2001, he joined wireless modem development division at GCT Research, and was a senior wireless communications engineer working on the development of various kinds of wireless modems including WCDMA, Bluetooth, 802.11abg Wireless LAN, and DMB. He is currently at WiMAX Program Office in Intel where he handles technical matters about wireless communications in Asia Pacific region. At the same time, he is now working towards the Ph.D. degree at the department of electronics convergence engineering, Kwangwoon University Graduate School in Seoul, Korea. His main research interests include OFDM, MIMO, and high speed wireless communications.

\section{Jin Young Kim}

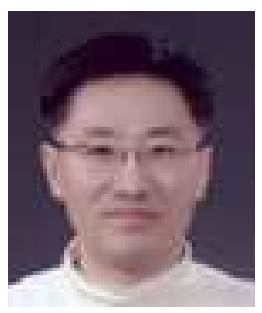

(S'91-M'95-SM'08) received the B.Sc., M.Sc., and Ph.D. degrees from the School of Electrical Engineering, Seoul National University (SNU), Seoul, Korea, in 1991, 1993, and 1998, respectively. He was Member of Research Staff at the Institute of New Media and Communications (INMC) and at the Inter-university Semiconductor Research Center (ISRC) of the SNU from 1994 to 1998. He was Postdoctoral Research Fellow at the Department of Electrical Engineering, Princeton University, NJ, U.S.A, from 1998 to 2000. He was Principal Member of Technical Staff at the Central Research and Development Center, SK Telecom, Korea, from 2000 to 2001. He is currently Associate Professor at the School of Electronics Engineering, Kwangwoon University, Seoul, Korea. Now, he has his sabbatical leave as Visiting Scientist at the LIDS (Laboratory of Information and Decision Systems), Massachusetts Institute of Technology (M.I.T), MA, U.S.A. His research interests include design and implementation of wireline/wireless multimedia communication systems for applications to spread-spectrum, cognitive radio, ultrawideband (UWB), space communication, optical communication and powerline communication systems with basis on modulation/demodulation, synchronization, channel coding, and detection/estimation theory. He received the Best Paper Awards from several academic conferences and societies including Jack Nebauer Best Systems Paper Award from IEEE VT Society (2001), the Award of Ministry of Information and Communication of Korea Government (1998), the Best Paper Award at APCC'00 (2000), the Best Paper Award at IEEE MoMuC'97 (1997), and the many other Best Paper Awards from conferences of IEEK'08, KITFE'08, KITS'08, and KITS'09 (20082009). He was listed in the Marquis Who's Who in the World, Marquis Who's Who in Science and Engineering, ABI and IBC throughout from 2001 to 2009 Editions. He is now Senior Member of IEEE, Regular Member of IET, IEICE, and Life Member of IEEK, KICS, KEES, KITFE, KITS, KIMST, KOSST and KOSBE. 